\title{
[BMIM][PF 6 Promotes the Synthesis of Halohydrin Esters from Diols Using Potassium Halides
}

\author{
Mireia Oromí-Farrús,* Jordi Eras,* Gemma Villorbina,* Mercè Torres,** \\ Veronica Llopis-MeSTRE, $* * *$ Tom Welton, $* * *$ and Ramon CANELA $* \dagger$ \\ *Department of Chemistry, University of Lleida, Av. Alcalde Rovira Roure, 191, Lleida 25198, Spain \\ **Department of Food Technology, University of Lleida, Av. Alcalde Rovira Roure, 191, Lleida 25198, Spain \\ ***Department of Chemistry, Imperial College of London, South Kensington Campus, \\ London SW7 2AZ, United Kingdom
}

\begin{abstract}
Haloesterification of diverse diols with various carboxylic acids was achieved using potassium halides (KX) as the only halide source in ionic liquids. The best yield was obtained in $[\mathrm{BMIM}]\left[\mathrm{PF}_{6}\right]$ when 1,2-octanediol, palmitic acid and $\mathrm{KBr}$ were used. This yield was $85 \%$ and the regioisomer with the bromine in primary position was present in a 75:25 ratio. The regioisomeric ratio could be improved using either $\mathrm{KCl}$ or some phenylcarboxylic acids. [BMIM] $\left[\mathrm{PF}_{6}\right]$ acts as both reaction media and catalyst of the reaction. To the best of our knowledge, this type of combined reaction using an ionic liquid is unprecedented. The other solvents tested did not lead either to the same yield or to the same regioisomeric ratio.
\end{abstract}

(Received April 7, 2008; Accepted June 16, 2008; Published October 10, 2008)

\section{Introduction}

Halohydrin esters are useful building blocks for the preparation of drugs, ${ }^{1}$ surfactants, ${ }^{2}$ epoxides, ${ }^{3}$ epichlorohydrins ${ }^{4}$ and nitriles. ${ }^{5}$ Some conventional halogenation methods use halogen sources which are pollutants, and generate hazardous halogenated byproducts. ${ }^{6,7}$ Moreover, methodologies to synthesize these halohydrins esters are usually expensive multi-step processes. ${ }^{8-16}$ In contrast, Eras et al. have developed a direct methodology to the stereospecific chlorohydrin ester formation. The method consist in one-pot esterification-chlorination reaction, in which chlorotrimethylsilane acts as a solvent, catalyzer and chlorine source. ${ }^{17}$ Trying to avoid the use of chlorotrimethylsilane, our work was focused on developing a new friendly one-pot halohydrin ester synthesis using potassium halides (KX) as halogen source in ionic liquids (ILs). ILs are a greener alternative to volatile organic solvents and their solvent properties may be easily controlled by altering the nature of their cations and anions. ${ }^{18}$ Moreover, ILs can act as a reaction catalyst. ${ }^{18-20}$ ILs have been used in many organic reactions and their applications are continuously growing..$^{21,22}$

Herein, we report the synthesis of halohydrin esters from different diols, carboxylic acids and $\mathrm{KX}$ in $[\mathrm{BMIM}]\left[\mathrm{PF}_{6}\right]$ as reaction media and catalyst (Fig. 1). To the best of our knowledge, this type of combined reaction is unprecedented. The same reaction using other ILs was also studied without observing an equivalent process.

† To whom correspondence should be addressed.

E-mail: canela@quimica.udl.cat

\section{Experimental}

\section{Reagents and chemicals}

1,2-Octanediol, 1-octanol, $\mathrm{KBr}, \mathrm{KCl}, \mathrm{KI}, \mathrm{KF}, 1,2$-pentanediol, butanone, 2-pentanone and 2-hexanone were purchased from Acros Organics, Barcelona, Spain. 1,2-Butanediol and benzoic acid were purchased from Fluka, Madrid, Spain. 18-Crown-6, 1,2-propanediol, 1,2-hexanediol, decanoic acid, ibuprofen, 1,1,3,3,5,5-hexamethyltrisiloxane (HMTSO), hexamethyldisiloxane (HMDSO), hexaethyldisiloxane (HEDSO), 1,1,1,3,5,5,5-heptamethyltrisiloxane (HpMTSO) were purchased from Sigma-Aldrich, Madrid, Spain. Cinnamic acid, 48\% aqueous $\mathrm{HF}$, $[\mathrm{BMIM}]\left[\mathrm{PF}_{6}\right],\left[\mathrm{BM}_{2} \mathrm{IM}\right]\left[\mathrm{PF}_{6}\right]$ and $[\mathrm{HMIM}]\left[\mathrm{PF}_{6}\right]$ were purchased from Merck, Barcelona, Spain. [HMIM][N(Tf $)_{2}$, $[\mathrm{HMIM}]\left[\mathrm{BF}_{4}\right], \quad[\mathrm{BMPy}]\left[\mathrm{N}(\mathrm{Tf})_{2}\right], \quad\left[\mathrm{BM}_{2} \mathrm{IM}\right]\left[\mathrm{N}(\mathrm{Tf})_{2}\right]$, [OMIM] $\left[\mathrm{N}(\mathrm{Tf})_{2}\right],[\mathrm{OMIM}]\left[\mathrm{BF}_{4}\right],[\mathrm{BPy}]\left[\mathrm{N}(\mathrm{Tf})_{2}\right],[\mathrm{BHIM}]\left[\mathrm{BF}_{4}\right]$, $[\mathrm{BDIM}]\left[\mathrm{BF}_{4}\right]$ and $[\mathrm{DBIM}]\left[\mathrm{BF}_{4}\right]$ were synthesized by metathesis in the Chemistry Department of the Imperial College of London.

\section{Apparatus}

NMR experiments were carried out in a MERCURYplus AS400 MHz with an AutoSwithable 4 Nucleus Probe from Varian. HRMS analyses were performed in an AVG AutoSpec Micromass with EI and magnetic sector analyzer.

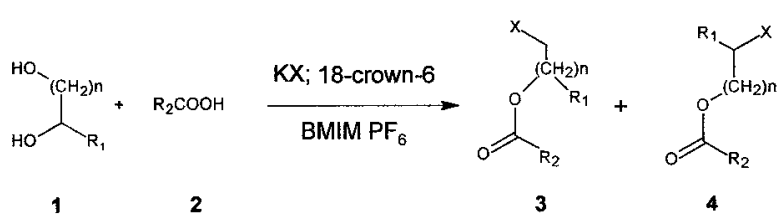

Fig. 1 Haloesterification of diols using ILs and $\mathrm{KX}$ as halide source. 


\section{General procedure}

A mixture of diol ( $0.8 \mathrm{mmol})$, acid $(0.4 \mathrm{mmol})$, anhydrous $\mathrm{KX}$ $(2.0 \mathrm{mmol})$ and ionic liquid $(0.8 \mathrm{mmol})$, conventional solvent $(0.5 \mathrm{~mL})$ or $\mathrm{HF}(0.014 \mathrm{mmol})$ was stirred at $100^{\circ} \mathrm{C}$ for $48 \mathrm{~h} .18$ Crown-6 ether $(0.013 \mathrm{mmol})$ was added at the beginning of the process when required. The reaction products were extracted with hexane, dried over $\mathrm{MgSO}_{4}$, filtered and concentrated in vасио. Reaction products were analyzed by ${ }^{1} \mathrm{H} \mathrm{NMR}$, with a $\mathrm{pw}=30^{\circ}$ and $\mathrm{dt}=10 \mathrm{~s}$, determining the crude yield by the integration of the most deshielded proton signals of each product. Reactions with a high percentage of a halohydrin ester were purified by column chromatography (silica gel H60, hexane/ethyl ether). The purified products were characterized by ${ }^{1} \mathrm{H}$ and ${ }^{13} \mathrm{C}$ NMR and HRMS. Each reaction was carried out more than once.

\section{Product characterization}

1-(Bromomethyl)heptyl palmitate (3a) and 2-bromooctyl palmitate (4a). HRMS (EI+) $m / z: 448.2926,446.2763$, 367.3839, 256.2867, 239.266, 111.1075; calculated 446.275942; found $446.276315,-0.8 \mathrm{ppm} ; 24 \mathrm{C}, 47 \mathrm{H}, 2 \mathrm{O}, 1 \mathrm{Br}$. 3a Regioisomer: ${ }^{1} \mathrm{H}$ NMR $\left(400 \mathrm{MHz}, \mathrm{CDCl}_{3}\right) ; \delta 4.98-4.88(\mathrm{~m}$, $1 \mathrm{H}), 3.44(\mathrm{dd}, J=10.94 \mathrm{~Hz}, 4.69 \mathrm{~Hz}, 1 \mathrm{H}), 3.35(\mathrm{dd}, J=10.94$, $5.47 \mathrm{~Hz}, 1 \mathrm{H}), 2.27(\mathrm{t}, J=7.42 \mathrm{~Hz}, 2 \mathrm{H}), 1.66-1.51(\mathrm{~m}, 4 \mathrm{H})$, $1.40-1.14(\mathrm{~m}, 32 \mathrm{H}), 0.81(\mathrm{t}, J=6.64 \mathrm{~Hz}, 6 \mathrm{H}) ;{ }^{13} \mathrm{C}$ NMR $(101$ $\left.\mathrm{MHz}, \mathrm{CDCl}_{3}\right) ; \delta 173.47,72.37,35.43,32.78,31.99,31.85$, $31.00,29.71-29.30,27.32,25.20,22.92,22.75,14.25$. 4a Regioisomer: ${ }^{1} \mathrm{H}$ NMR (400 MHz, $\left.\mathrm{CDCl}_{3}\right) ; \delta 4.30-4.17(\mathrm{~m}$, 2H), $4.09-3.99(\mathrm{~m}, 1 \mathrm{H}), 1.86-1.65(\mathrm{~m}, 2 \mathrm{H}), 0.81(\mathrm{t}, J=6.64$ $\mathrm{Hz}, 6 \mathrm{H}) ;{ }^{13} \mathrm{C}$ NMR $\left(101 \mathrm{MHz}, \mathrm{CDCl}_{3}\right) ; \delta 173.55,67.84,51.95$, $34.70,34.53,32.15,31.94,31.82,28.85,25.16,22.80,14.33$. All other NMR signals are hidden by the signals of the $\mathbf{3 a}$ regioisomer.

1-(Bromomethyl)pentyl palmitate $(\mathbf{3 b})$ and 2-bromohexyl palmitate (4b). HRMS (EI $\left.{ }^{+}\right) \quad m / z:$ 418.2474, 292.9824, 256.2394, 239.2388, 93.08591; calculated 418.2501; found 418.2474; 22C, 43H, 2O, 1Br. 3b Regioisomer: ${ }^{1} \mathrm{H}$ NMR (400 $\left.\mathrm{MHz}, \mathrm{CDCl}_{3}\right) ; \delta 5.06-4.96(\mathrm{~m}, 1 \mathrm{H}), 3.51(\mathrm{dd}, J=10.94,4.69$ $\mathrm{Hz}, 1 \mathrm{H}), 3.43(\mathrm{dd}, J=10.94,5.47 \mathrm{~Hz}, 1 \mathrm{H}), 2.34(\mathrm{t}, J=6.25 \mathrm{~Hz}$, $2 \mathrm{H}), 1.73-1.53(\mathrm{~m}, 4 \mathrm{H}), 1.43-1.22(\mathrm{~m}, 28 \mathrm{H}), 0.94-0.89(\mathrm{~m}$, $3 \mathrm{H}), 0.88$ (t, $J=7.03 \mathrm{~Hz}, 3 \mathrm{H}) ;{ }^{13} \mathrm{C}$ NMR $\left(101 \mathrm{MHz}, \mathrm{CDCl}_{3}\right) ; \delta$ 173.53, 72.37, 35.14, 34.55, 32.47, 32.14, 29.90 - 29.34, 27.43, 25.15, 22.87, 22.29, 14.32, 14.11. 4b Regioisomer: ${ }^{1} \mathrm{H}$ NMR $\left(400 \mathrm{MHz}, \mathrm{CDCl}_{3}\right) ; \delta 4.36-4.26(\mathrm{~m}, 2 \mathrm{H}), 4.16-4.05(\mathrm{~m}, 1 \mathrm{H})$, $2.34(\mathrm{t}, J=7.42 \mathrm{~Hz}, 2 \mathrm{H}), 1.93-1.73(\mathrm{~m}, 2 \mathrm{H}), 0.88(\mathrm{t}, J=7.03$ $\mathrm{Hz}, 3 \mathrm{H}) ;{ }^{13} \mathrm{C}$ NMR $\left(101 \mathrm{MHz}, \mathrm{CDCl}_{3}\right) ; \delta 173.50,67.84,51.95$, $34.66,34.40,31.81,31.12,25.26,22.90,22.62,14.32,11.64$. All other NMR signals are hidden by the signals of the $\mathbf{3 b}$ regioisomer.

1-(Bromomethyl)butyl palmitate (3c) and 2-bromopentyl palmitate (4c). HRMS (EI $\left.{ }^{+}\right) \mathrm{m} / z:$ 404.2324, 292.9824, 256.2391, 239.2365, 69.0702; calculated 404.2358; found 404.2324; 21C, 41H, 2O, 1Br. 3c Regioisomer: ${ }^{1} \mathrm{H}$ NMR (400 $\left.\mathrm{MHz}, \mathrm{CDCl}_{3}\right) ; \delta 4.99-4.90(\mathrm{~m}, 1 \mathrm{H}), 3.44(\mathrm{dd}, J=10.75,4.89$ $\mathrm{Hz}, 1 \mathrm{H}), 3.35$ (dd, $J=10.75,5.37 \mathrm{~Hz}, 1 \mathrm{H}), 2.27(\mathrm{t}, J=7.33 \mathrm{~Hz}$, $2 \mathrm{H}), 1.65-1.52(\mathrm{~m}, 4 \mathrm{H}), 1.33-1.12(\mathrm{~m}, 26 \mathrm{H}), 0.87(\mathrm{t}, J=7.33$ $\mathrm{Hz}, 3 \mathrm{H}), 0.81(\mathrm{t}, J=6.84 \mathrm{~Hz}, 3 \mathrm{H}) ;{ }^{13} \mathrm{C} \mathrm{NMR}(101 \mathrm{MHz}$, $\left.\mathrm{CDCl}_{3}\right) ; \delta 178.19,69.58,33.65,33.41,33.22,32.91,29.28$ 28.05, 24.02, 23.68, 21.68, 13.10. 4c Regioisomer: ${ }^{1} \mathrm{H}$ NMR $\left(400 \mathrm{MHz}, \mathrm{CDCl}_{3}\right) ; \delta 4.29-4.17(\mathrm{~m}, 1 \mathrm{H}), 4.10-4.01(\mathrm{~m}, 2 \mathrm{H})$, $2.27(\mathrm{t}, J=7.82 \mathrm{~Hz}, 2 \mathrm{H}), 1.84-1.66(\mathrm{~m}, 2 \mathrm{H}) ;{ }^{13} \mathrm{C}$ NMR $(101$ $\left.\mathrm{MHz}, \mathrm{CDCl}_{3}\right) ; \delta 178.19,68.21,50.43,33.42,33.22,30.94$, $25.95,24.02,23.91,17.38,13.10$. All other NMR signals are hidden by the signals of the $\mathbf{3 c}$ regioisomer.

1-(Bromomethyl)propyl palmitate (3d) and 2-bromobutyl palmitate (4d). HRMS (EI $\left.{ }^{+}\right) \quad m / z: 390.2165,257.2460$, 239.2350, 55.05238; calculated 390.2197; found 390.2165; 20C, 39H, 2O, 1Br. 3d Regioisomer: ${ }^{1} \mathrm{H}$ NMR (400 MHz, $\left.\mathrm{CDCl}_{3}\right) ; \delta$ $4.93-4.83(\mathrm{~m}, 1 \mathrm{H}), 3.44(\mathrm{dd}, J=10.94,4.69 \mathrm{~Hz}, 1 \mathrm{H}), 3.37$ (dd, $J=10.94,5.47 \mathrm{~Hz}, 1 \mathrm{H}), 2.27(\mathrm{t}, J=7.42 \mathrm{~Hz}, 2 \mathrm{H}), 1.80-1.62$ $(\mathrm{m}, 2 \mathrm{H}), 1.62-1.53(\mathrm{~m}, 2 \mathrm{H}), 1.31-1.15(\mathrm{~m}, 24 \mathrm{H}), 0.86(\mathrm{t}, J=$ $7.42 \mathrm{~Hz}, 3 \mathrm{H}), 0.81(\mathrm{t}, J=7.03 \mathrm{~Hz}, 3 \mathrm{H}) ;{ }^{13} \mathrm{C} \mathrm{NMR}(101 \mathrm{MHz}$, $\left.\mathrm{CDCl}_{3}\right) ; \delta 173.53,73.54,34.64,34.03,32.14,29.90-29.34$, 25.26, 25.15, 22.91, 14.33, 9.61. 4d Regioisomer: ${ }^{1} \mathrm{H}$ NMR $\left(400 \mathrm{MHz}, \mathrm{CDCl}_{3}\right) ; \delta 4.30-4.17(\mathrm{~m}, 2 \mathrm{H}), 4.06-3.93(\mathrm{~m}, 1 \mathrm{H})$, $2.28(\mathrm{t}, J=7.42 \mathrm{~Hz}, 2 \mathrm{H}), 1.96-1.81(\mathrm{~m}, 2 \mathrm{H}), 1.00(\mathrm{t}, J=7.03$ $\mathrm{Hz}, 3 \mathrm{H}), 0.81(\mathrm{t}, J=7.03 \mathrm{~Hz}, 3 \mathrm{H}) ;{ }^{13} \mathrm{C}$ NMR $(101 \mathrm{MHz}$, $\left.\mathrm{CDCl}_{3}\right) ; \delta 173.00,67.49,53.58,34.39,25.83,14.33,9.61$. All other NMR signals are hidden by the signals of the $\mathbf{3 d}$ regioisomer.

1-(Bromomethyl)ethyl palmitate $(\mathbf{3 e})$ and 2-bromopropyl palmitate (4e). HRMS (EI $\left.{ }^{+}\right) \mathrm{m} / z$ : 376.1985, 255.2301, 239.2301, 120.9642; calculated 376.1993; found 376.1985; 19C, 37H, 2O, 1Br. 3e Regioisomer: ${ }^{1} \mathrm{H}$ NMR $\left(400 \mathrm{MHz}, \mathrm{CDCl}_{3}\right) ; \delta$ $5.07-4.97(\mathrm{~m}, 1 \mathrm{H}), 3.45-3.30(\mathrm{~m}, 2 \mathrm{H}), 2.25(\mathrm{t}, J=7.42 \mathrm{~Hz}$, $2 \mathrm{H}), 1.61-1.52(\mathrm{~m}, 2 \mathrm{H}), 1.28(\mathrm{~d}, J=6.25 \mathrm{~Hz}, 3 \mathrm{H}), 1.26-1.14$ $(\mathrm{m}, 24 \mathrm{H}), 0.81(\mathrm{t}, J=7.03 \mathrm{~Hz}, 3 \mathrm{H}) ;{ }^{13} \mathrm{C}$ NMR $(101 \mathrm{MHz}$, $\left.\mathrm{CDCl}_{3}\right) ; \delta 173.29,68.92,35.53,34.37,32.14,29.90-29.31$, 25.14, 22.68, 18.89, 14.33. 4e Regioisomer: ${ }^{1} \mathrm{H}$ NMR (400 $\left.\mathrm{MHz}, \mathrm{CDCl}_{3}\right) ; \delta 4.25-4.18(\mathrm{~m}, 1 \mathrm{H}), 4.17-4.09(\mathrm{~m}, 2 \mathrm{H}), 2.28$ $(\mathrm{t}, J=7.42 \mathrm{~Hz}, 2 \mathrm{H}), 1.63(\mathrm{~d}, J=6.64 \mathrm{~Hz}, 3 \mathrm{H}), 0.81(\mathrm{t}, J=7.03$ $\mathrm{Hz}, 3 \mathrm{H}) ;{ }^{13} \mathrm{C}$ NMR $\left(101 \mathrm{MHz}, \mathrm{CDCl}_{3}\right) ; \delta 173.48,69.19,45.08$, $34.65,25.19,22.91$. All other NMR signals are hidden by the signals of the $\mathbf{3 e}$ regioisomer.

3-Bromo-1-methylpropyl palmitate $(\mathbf{3 f})$ and 3-bromobutyl palmitate (4f). HRMS $\left(\mathrm{EI}^{+}\right) \mathrm{m} / \mathrm{z}: 390.2146,256.2387,239.2359$, 136.9795, 56.06384; calculated 390.2158; found 390.2146; 20C, 39H, 2O, 1Br. 3f Regioisomer: ${ }^{1} \mathrm{H}$ NMR (400 MHz, $\mathrm{CDCl}_{3}$ ); $\delta$ $5.12-4.98(\mathrm{~m}, 1 \mathrm{H}), 3.46-3.31(\mathrm{~m}, 2 \mathrm{H}), 2.29(\mathrm{t}, J=7.42 \mathrm{~Hz}$, $2 \mathrm{H}), 2.22-2.10(\mathrm{~m}, 1 \mathrm{H}), 2.09-1.98(\mathrm{~m}, 1 \mathrm{H}), 1.67-1.58(\mathrm{~m}$, 2H), $1.37-1.22(\mathrm{~m}, 27 \mathrm{H}), 0.88(\mathrm{t}, J=7.03 \mathrm{~Hz}, 3 \mathrm{H}) ;{ }^{13} \mathrm{C} \mathrm{NMR}$ $\left(101 \mathrm{MHz}, \mathrm{CDCl}_{3}\right) ; \delta 173.50,69.13,39.24,34.81,32.14,29.90$ - 28.84, 25.26, 22.91, 20.06, 14.33. 4f Regioisomer: ${ }^{1} \mathrm{H}$ NMR $\left(400 \mathrm{MHz}, \mathrm{CDCl}_{3}\right) ; \delta 4.30-4.22(\mathrm{~m}, 1 \mathrm{H}), 4.22-4.17(\mathrm{~m}, 2 \mathrm{H})$, $2.09-1.98(\mathrm{~m}, 1 \mathrm{H}), 1.75(\mathrm{~d}, J=6.64 \mathrm{~Hz}, 3 \mathrm{H}) ;{ }^{13} \mathrm{C}$ NMR $(101$ $\left.\mathrm{MHz}, \mathrm{CDCl}_{3}\right) ; \delta 177.25,62.31,46.98,39.63,34.30,32.15$, $26.77,26.15,23.46$. All other NMR signals are hidden by the signals of the $3 \mathbf{f}$ regioisomer.

1-(Bromomethyl)heptyl acetate $(3 \mathrm{~g})$ and 2-bromooctyl acetate (4g). 3g Regioisomer: ${ }^{1} \mathrm{H}$ NMR $\left(400 \mathrm{MHz}, \mathrm{CDCl}_{3}\right) ; \delta 5.04-$ $4.96(\mathrm{~m}, 1 \mathrm{H}), 3.51(\mathrm{dd}, J=10.75,4.89 \mathrm{~Hz}, 1 \mathrm{H}), 3.43(\mathrm{dd}, J=$ 10.75, $5.37 \mathrm{~Hz}, 1 \mathrm{H}), 2.11(\mathrm{~s}, 3 \mathrm{H}), 1.75-1.49(\mathrm{~m}, 2 \mathrm{H}), 1.48-$ $1.18(\mathrm{~m}, 8 \mathrm{H}), 0.90(\mathrm{t}, J=6.84 \mathrm{~Hz}, 3 \mathrm{H}) ;{ }^{13} \mathrm{C} \mathrm{NMR}(101 \mathrm{MHz}$, $\left.\mathrm{CDCl}_{3}\right) ; \delta 172.10,72.83,36.40,31.87,31.76,27.90$ - 22.61, 21.16, 14.11. 4g Regioisomer: ${ }^{1} \mathrm{H}$ NMR (400 MHz, $\left.\mathrm{CDCl}_{3}\right) ; \delta$ $4.36-4.25(\mathrm{~m}, 2 \mathrm{H}), 4.16-4.07(\mathrm{~m}, 1 \mathrm{H}), 2.07(\mathrm{~s}, 3 \mathrm{H}), 1.92$ $1.73(\mathrm{~m}, 2 \mathrm{H}) ;{ }^{13} \mathrm{C}$ NMR $\left(101 \mathrm{MHz}, \mathrm{CDCl}_{3}\right) ; \delta 170.39,67.68$, $51.79,35.26,21.05$. All other NMR signals are hidden by the signals of the $\mathbf{3 g}$ regioisomer.

1-(Bromomethyl)heptyl decanoate (3h) and 2-bromooctyl decanoate (4h). 3h Regioisomer: ${ }^{1} \mathrm{H} \mathrm{NMR}\left(400 \mathrm{MHz}, \mathrm{CDCl}_{3}\right)$; $\delta 4.98-4.87(\mathrm{~m}, 1 \mathrm{H}), 3.44(\mathrm{dd}, J=10.94,4.69 \mathrm{~Hz}, 1 \mathrm{H}), 3.36$ $(\mathrm{dd}, J=10.94,5.47 \mathrm{~Hz}, 1 \mathrm{H}), 2.26(\mathrm{t}, J=7.82 \mathrm{~Hz}, 2 \mathrm{H}), 1.67$ $1.41(\mathrm{~m}, 4 \mathrm{H}), 1.31-1.11(\mathrm{~m}, 20 \mathrm{H}), 0.81(\mathrm{t}, J=6.64 \mathrm{~Hz}, 6 \mathrm{H})$; ${ }^{13} \mathrm{C}$ NMR $\left(101 \mathrm{MHz}, \mathrm{CDCl}_{3}\right) ; \delta 173.53,72.38,35.43,32.78-$ $31.80,29.64,29.52$ - 28.84, 27.32, 25.29, 25.26, 14.24. 4h Regioisomer: ${ }^{1} \mathrm{H}$ NMR (400 MHz, $\left.\mathrm{CDCl}_{3}\right) ; \delta 4.29-4.17(\mathrm{~m}$, $2 \mathrm{H}), 4.10-3.99(\mathrm{~m}, 1 \mathrm{H}), 2.25(\mathrm{t}, J=6.64 \mathrm{~Hz}, 2 \mathrm{H}), 1.85-1.65$ $(\mathrm{m}, 2 \mathrm{H}) ;{ }^{13} \mathrm{C}$ NMR $\left(101 \mathrm{MHz}, \mathrm{CDCl}_{3}\right) ; \delta 173.49,67.84,51.99$, $34.66,32.07,25.16,22.88,14.31$. All other NMR signals are 
hidden by the signals of the $\mathbf{3 h}$ regioisomer.

1-(Bromomethyl)heptyl benzoate (3i). ${ }^{1} \mathrm{H}$ NMR (400 MHz, $\left.\mathrm{CDCl}_{3}\right) ; \delta 8.00(\mathrm{dd}, 2 \mathrm{H}), 7.58-7.48(\mathrm{~m}, 3 \mathrm{H}), 5.22-5.14(\mathrm{~m}$, $1 \mathrm{H}), 3.64-3.58(\mathrm{~m}, 2 \mathrm{H}), 1.81-1.71(\mathrm{~m}, 2 \mathrm{H}), 1.42-1.09(\mathrm{~m}$, $8 \mathrm{H}), 0.80$ (t, $J=6.35 \mathrm{~Hz}, 3 \mathrm{H}) ;{ }^{13} \mathrm{C} \mathrm{NMR}\left(101 \mathrm{MHz}, \mathrm{CDCl}_{3}\right) ; \delta$ $167.74,132.71,131.57,130.10,128.35,75.11,36.47,32.05-$ 26.31, 22.54, 14.11.

1-(Bromomethyl)heptyl cinnamate $(3 \mathbf{j})$ and 2-bromooctyl cinnamate (4j). 3j Regioisomer: ${ }^{1} \mathrm{H}$ NMR $\left(400 \mathrm{MHz}, \mathrm{CDCl}_{3}\right) ; \delta$ $7.68(\mathrm{~d}, 1 \mathrm{H}), 7.51-7.39(\mathrm{~m}, 2 \mathrm{H}), 7.37-7.26(\mathrm{~m}, 3 \mathrm{H}), 6.42(\mathrm{~d}$, 1H), $5.11-5.02(\mathrm{~m}, 1 \mathrm{H}), 3.51$ (dd, $J=10.81,4.64 \mathrm{~Hz}, 1 \mathrm{H}), 3.44$ (dd, $J=10.81,5.25 \mathrm{~Hz}, 1 \mathrm{H}), 1.73-1.65(\mathrm{~m}, 2 \mathrm{H}), 1.44-1.11$ $(\mathrm{m}, 8 \mathrm{H}), 0.80(\mathrm{t}, J=6.77 \mathrm{~Hz}, 3 \mathrm{H}) ;{ }^{13} \mathrm{C} \mathrm{NMR}\left(101 \mathrm{MHz}, \mathrm{CDCl}_{3}\right)$; $\delta 168.51,144.69,134.35,130.36,129.11,128.47,118.44$, 72.42, 36.49, 32.02 - 22.61, 14.11. 4j Regioisomer: ${ }^{1} \mathrm{H}$ NMR $\left(400 \mathrm{MHz}, \mathrm{CDCl}_{3}\right) ; \delta 7.63(\mathrm{~d}, 1 \mathrm{H}), 6.38(\mathrm{~d}, 1 \mathrm{H}), 4.42-4.33(\mathrm{~m}$, 2H), $4.17-4.08(\mathrm{~m}, 1 \mathrm{H}), 1.91-1.73(\mathrm{~m}, 2 \mathrm{H}) ;{ }^{13} \mathrm{C}$ NMR $(101$ $\left.\mathrm{MHz}, \mathrm{CDCl}_{3}\right) ; \delta 166.03,145.53,134.71,130.88,129.10$, $127.83,117.40,68.82,50.77$. All other NMR signals are hidden by the signals of the $\mathbf{4} \mathbf{j}$ regioisomer.

1-(Bromomethyl)heptyl ibuprofenate $(\mathbf{3 k})$ and 2-bromooctyl ibuprofenate (4k). 3k Regioisomer: ${ }^{1} \mathrm{H}$ NMR $(400 \mathrm{MHz}$, $\left.\mathrm{CDCl}_{3}\right) ; \delta 7.21(\mathrm{~d}, J=8.31 \mathrm{~Hz}, 2 \mathrm{H}), 7.09(\mathrm{~d}, J=8.31 \mathrm{~Hz}, 2 \mathrm{H})$, $5.01-4.93(\mathrm{~m}, 1 \mathrm{H}), 3.75-3.70(\mathrm{~m}, 1 \mathrm{H}), 3.41(\mathrm{dd}, J=10.75$, $5.37 \mathrm{~Hz}, 1 \mathrm{H}), 3.36(\mathrm{dd}, J=10.75,5.37 \mathrm{~Hz}, 1 \mathrm{H}), 2.44(\mathrm{~d}, J=$ $6.84 \mathrm{~Hz}, 2 \mathrm{H}), 1.89-1.78(\mathrm{~m}, 1 \mathrm{H}), 1.61-1.52(\mathrm{~m}, 2 \mathrm{H}), 1.52(\mathrm{~d}$, $J=6.84 \mathrm{~Hz}, 3 \mathrm{H}), 1.36-1.16(\mathrm{~m}, 8 \mathrm{H}), 0.90(\mathrm{~d}, J=6.35 \mathrm{~Hz}, 6 \mathrm{H})$, $0.89(\mathrm{t}, J=6.84 \mathrm{~Hz}, 3 \mathrm{H}) ;{ }^{13} \mathrm{C}$ NMR $\left(101 \mathrm{MHz}, \mathrm{CDCl}_{3}\right) ; \delta$ $174.98,140.55,136.87,129.15,126.84,75.51,45.12,45.07$, $36.43,32.06,31.79,30.15,22.52-27.95,22.33,18.21,14.11$. 4k Regioisomer: ${ }^{1} \mathrm{H}$ NMR (400 MHz, $\left.\mathrm{CDCl}_{3}\right) ; \delta 4.32-4.25(\mathrm{~m}$, 2H), $4.09-3.99(\mathrm{~m}, 1 \mathrm{H}), 3.75-3.70(\mathrm{~m}, 1 \mathrm{H}) ;{ }^{13} \mathrm{C}$ NMR $(101$ $\left.\mathrm{MHz}, \mathrm{CDCl}_{3}\right) ; \delta 170.46,137.63,129.08,127.21,68.81,50.77$, $44.48,35.60$. All other NMR signals are hidden by the signals of the $\mathbf{3 k}$ regioisomer.

1-(Chloromethyl)heptyl palmitate (3l) and 2-chlorooctyl palmitate $(4 l)$. HRMS $\left(\mathrm{EI}^{+}\right) \mathrm{m} / \mathrm{z}: 402.3271,323.2540,256.2383$, 239.2384, 110.1094; calculated 402.3278; found 402.3271, -0.7 ppm; 24C, 47H, 2O, 1Cl. 3l Regioisomer: ${ }^{1} \mathrm{H}$ NMR $(400 \mathrm{MHz}$, $\left.\mathrm{CDCl}_{3}\right) ; \delta 5.02-4.92(\mathrm{~m}, 1 \mathrm{H}), 3.57(\mathrm{dd}, J=11.72,4.30 \mathrm{~Hz}$, $1 \mathrm{H}), 3.49(\mathrm{dd}, J=11.33,5.47 \mathrm{~Hz}, 1 \mathrm{H}), 2.26(\mathrm{t}, J=7.82 \mathrm{~Hz}$, $2 \mathrm{H}), 1.65-1.52(\mathrm{~m}, 4 \mathrm{H}), 1.32-1.13(\mathrm{~m}, 32 \mathrm{H}), 0.80(\mathrm{t}, J=7.03$ $\mathrm{Hz}, 6 \mathrm{H}) ;{ }^{13} \mathrm{C}$ NMR $\left(101 \mathrm{MHz}, \mathrm{CDCl}_{3}\right) ; \delta 173.57,72.81,46.02$, $34.66,32.14,31.85,31.78,29.91-29.21,25.26,25.23,22.91$, 22.75, 14.33, 14.25. 4l Regioisomer: ${ }^{1} \mathrm{H}$ NMR (400 MHz, $\left.\mathrm{CDCl}_{3}\right) ; \delta 4.21-4.10(\mathrm{~m}, 2 \mathrm{H}), 4.03-3.92(\mathrm{~m}, 1 \mathrm{H}), 2.28(\mathrm{t}, J=$ $7.03 \mathrm{~Hz}, 2 \mathrm{H}) ;{ }^{13} \mathrm{C}$ NMR $\left(101 \mathrm{MHz}, \mathrm{CDCl}_{3}\right) ; \delta 173.59,67.75$, $66.88,46.05,34.62,32.10,31.87,31.76,25.29,24.44,22.87$, $22.77,14.35,14.23$. All other NMR signals are hidden by the signals of the $\mathbf{3 1}$ regioisomer.

\section{Results and Discussion}

Table 1 shows the diverse ILs studied for the reaction of 1,2octanediol, palmitic acid and $\mathrm{KBr} . \quad \alpha, \beta$-Bromooctyl palmitates $3 \mathbf{a}$ and $\mathbf{4 a}$, were obtained in $75 \%$ of crude yield (43\% of isolated yield) when $[\mathrm{BMIM}]\left[\mathrm{PF}_{6}\right]$ was used. This ionic liquid led to a 3a:4a regioisomeric ratio of 64:36. Although bromohydrin esters with higher proportions of the regioisomer $\mathbf{3 a}$ were also formed with all the ILs containing the $\left[\mathrm{N}(\mathrm{Tf})_{2}\right]$ anion, crude yields were always much lower $(4-30 \%)$. When the anion was $\left[\mathrm{BF}_{4}\right]$, a slight formation of bromohydrin esters appeared with $[\mathrm{OMIM}]\left[\mathrm{BF}_{4}\right]$ and $[\mathrm{BDIM}]\left[\mathrm{BF}_{4}\right]$. The rest of the ILs only led to mixtures of 1,2-octanediol esters.
Table 1 Bromoesterification with 1,2-octanediol, palmitic acid and potassium bromide in different ionic liquids

\begin{tabular}{lcc}
\hline \multirow{2}{*}{ Ionic liquid } & \multicolumn{2}{c}{ Product $(\mathbf{3}, \mathbf{4})$} \\
\cline { 2 - 3 } & \% Crude yield & $\mathbf{3 : 4}$ ratio \\
\hline$[\mathrm{BMIM}]\left[\mathrm{PF}_{6}\right]$ & 75 & $64: 36$ \\
{$[\mathrm{HMIM}]\left[\mathrm{N}(\mathrm{Tf})_{2}\right]$} & 13 & $77: 23$ \\
{$[\mathrm{OMIM}]\left[\mathrm{N}(\mathrm{Tf})_{2}\right]$} & 5 & $60: 40$ \\
{$\left[\mathrm{BM} \mathrm{BIIM}_{2} \mathrm{M}(\mathrm{Nf})_{2}\right]$} & 30 & $100: 0$ \\
{$[\mathrm{BPy}]\left[\mathrm{N}(\mathrm{Tf})_{2}\right]$} & 4 & $75: 25$ \\
{$[\mathrm{BMPy}]\left[\mathrm{N}(\mathrm{Tf})_{2}\right]$} & 8 & $63: 37$ \\
{$\left[\mathrm{~B}_{2} \mathrm{IM}\right]\left[\mathrm{BF}_{4}\right]$} & - & - \\
{$[\mathrm{HMIM}]\left[\mathrm{BF}_{4}\right]$} & - & - \\
{$[\mathrm{OMIM}]\left[\mathrm{BF}_{4}\right]$} & 4 & $100: 0$ \\
{$\left[\mathrm{BM} \mathrm{BM}_{2} \mathrm{IM}\right]\left[\mathrm{BF}_{4}\right]$} & - & - \\
{$[\mathrm{BDIM}]\left[\mathrm{BF}_{4}\right]$} & 5 & $80: 20$ \\
{$[\mathrm{BPy}]\left[\mathrm{BF}_{4}\right]$} & - & - \\
\hline
\end{tabular}

Then, in order to improve the yield using $[\mathrm{BMIM}]\left[\mathrm{PF}_{6}\right]$, the reaction was studied in the presence of a crown-ether, 18-crown6 , a $\mathrm{K}^{+}$scavenger. The potassium complexation could increase the bromine reactivity due to either the ion dissociation or the increase of basic complex properties. ${ }^{23}$ When this ether was used, $\alpha, \beta$-bromooctyl palmitate yield increased from 43 to $85 \%$ (Table 2 , entry $\mathbf{a}$ ), increasing also the $\mathbf{3 a} \mathbf{a} \mathbf{4 a}$ regioisomeric ratio to $75: 25$. These results are in concordance with those described by Eras et al. in which the halohydrin ester having the halogen in the primary position was mainly formed. ${ }^{17}$ Apart from $\alpha, \beta$-bromooctyl palmitates, small amounts of hydroxyoctyl palmitates, 1,2-octanediol dipalmitates and bromooctanols were also obtained. However, the bromohydrin esters (3a, 4a) are easily purified using silica column chromatography. Although deeper studies could improve the yield and regioselectivity of the reaction, the described results are similar to or better than others described in the literature for preparing similar compounds. ${ }^{8,10-14,16,24}$

The reaction was carried out using either $\left[\mathrm{BM}_{2} \mathrm{IM}\right]\left[\mathrm{PF}_{6}\right]$ or $[\mathrm{HMIM}]\left[\mathrm{PF}_{6}\right]$ to study the effect of the $\left[\mathrm{PF}_{6}\right]$ anion in this bromination-esterification reaction. When $\left[\mathrm{BM}_{2} \mathrm{IM}\right]\left[\mathrm{PF}_{6}\right]$ was used, a $76 \%$ crude yield of the regioisomers $\mathbf{3 a}$ and $\mathbf{4 a}$ was obtained with a 3a:4a regioisomeric ratio of 70:30, yield and regioselectivity lower than the one obtained using $[\mathrm{BMIM}]\left[\mathrm{PF}_{6}\right]$. For $[\mathrm{HMIM}]\left[\mathrm{PF}_{6}\right]$, a higher yield was obtained. However, the 3a:4a regioisomeric ratio was worse: $65: 35$. These results could indicate that the anion has the major effect in the performance of the reaction but that the cation also influences the reaction yield and its regioselectivity.

Since some degradation of $\left[\mathrm{PF}_{6}\right]$ anion could take place with the consequent formation of $\mathrm{HF}$, the reactions with $\mathrm{HF}$ without the IL have been tested. Although some reaction occurs (19\% of bromohydrin esters of crude yield) it is not the same as in the $\mathrm{IL}$, so the IL is needed to achieve the bromination-esterification reaction.

The reactions in some conventional organic solvents (acetone, tert-butyl methyl ether, dimethylformamide acetonitrile, butanone, 2-pentanone and 2-hexanone and some alkyl silyl ethers: HMTSO, HMDSO, MEDSO, HpMTSO) were also studied. In most of these organic solvents, no formation of $\alpha, \beta$ bromooctyl palmitates was observed. Only when HMTSO was used, a 3\% quantity of bromohydrin esters was formed. We have also tested the reaction in HMTSO with the presence of 18-crown-6 ether, but no increase of the yield was observed.

Our interest was then focused on studying the scope of this 
Table 2 Haloesterification with different diols, carboxylic acids and potassium halides and 18-crown-6 in [BMIM] $\left[\mathrm{PF}_{6}\right]$

\begin{tabular}{|c|c|c|c|c|c|c|}
\hline \multirow{3}{*}{ Entry } & \multicolumn{3}{|c|}{ Reagent } & \multirow{3}{*}{$X$} & \multicolumn{2}{|c|}{ Product $(\mathbf{3}, \mathbf{4})$} \\
\hline & \multicolumn{2}{|c|}{ Alcohol (1) } & \multirow{2}{*}{$\begin{array}{c}\text { Acid (2) } \\
R_{2}\end{array}$} & & \multirow{2}{*}{$\begin{array}{l}\% \text { Crude yield } \\
\text { (isolated yield) }\end{array}$} & \multirow{2}{*}{$3: 4$ ratio } \\
\hline & $n$ & $R_{1}$ & & & & \\
\hline $\mathbf{a}$ & 1 & $\mathrm{CH}_{3}\left(\mathrm{CH}_{2}\right)_{5}$ & $\mathrm{CH}_{3}\left(\mathrm{CH}_{2}\right)_{14}$ & $\mathrm{Br}$ & $90(85)$ & $75: 25$ \\
\hline b & 1 & $\mathrm{CH}_{3}\left(\mathrm{CH}_{2}\right)_{3}$ & $\mathrm{CH}_{3}\left(\mathrm{CH}_{2}\right)_{14}$ & $\mathrm{Br}$ & $75(60)$ & $67: 33$ \\
\hline c & 1 & $\mathrm{CH}_{3}\left(\mathrm{CH}_{2}\right)_{2}$ & $\mathrm{CH}_{3}\left(\mathrm{CH}_{2}\right)_{14}$ & $\mathrm{Br}$ & $82(60)$ & $70: 30$ \\
\hline d & 1 & $\mathrm{CH}_{3} \mathrm{CH}_{2}$ & $\mathrm{CH}_{3}\left(\mathrm{CH}_{2}\right)_{14}$ & $\mathrm{Br}$ & $62(53)$ & $65: 35$ \\
\hline $\mathbf{e}$ & 1 & $\mathrm{CH}_{3}$ & $\mathrm{CH}_{3}\left(\mathrm{CH}_{2}\right)_{14}$ & $\mathrm{Br}$ & $57(47)$ & $72: 28$ \\
\hline f & 2 & $\mathrm{CH}_{3}$ & $\mathrm{CH}_{3}\left(\mathrm{CH}_{2}\right)_{14}$ & $\mathrm{Br}$ & $55(41)$ & $66: 34$ \\
\hline g & 1 & $\mathrm{CH}_{3}\left(\mathrm{CH}_{2}\right)_{5}$ & $\mathrm{CH}_{3}$ & $\mathrm{Br}$ & $82(-)$ & $62: 38$ \\
\hline h & 1 & $\mathrm{CH}_{3}\left(\mathrm{CH}_{2}\right)_{5}$ & $\mathrm{CH}_{3}\left(\mathrm{CH}_{2}\right.$ & $\mathrm{Br}$ & $84(43)$ & $67: 33$ \\
\hline i & 1 & $\mathrm{CH}_{3}\left(\mathrm{CH}_{2}\right)_{5}$ & & $\mathrm{Br}$ & $12(-)$ & 100:0 \\
\hline $\mathbf{j}$ & 1 & $\mathrm{CH}_{3}\left(\mathrm{CH}_{2}\right)_{5}$ & & $\mathrm{Br}$ & $48(-)$ & $58: 42$ \\
\hline k & 1 & $\mathrm{CH}_{3}\left(\mathrm{CH}_{2}\right)_{5}$ & & $\mathrm{Br}$ & $57(-)$ & $80: 20$ \\
\hline l & 1 & $\mathrm{CH}_{3}\left(\mathrm{CH}_{2}\right)_{5}$ & $\mathrm{CH}_{3}\left(\mathrm{CH}_{2}\right)_{14}$ & $\mathrm{Cl}$ & $86(55)$ & $80: 20$ \\
\hline m & 1 & $\mathrm{CH}_{3}\left(\mathrm{CH}_{2}\right)_{5}$ & $\mathrm{CH}_{3}\left(\mathrm{CH}_{2}\right)_{14}$ & I & nd & - \\
\hline $\mathbf{n}$ & 1 & $\mathrm{CH}_{3}\left(\mathrm{CH}_{2}\right)_{5}$ & $\mathrm{CH}_{3}\left(\mathrm{CH}_{2}\right)_{14}$ & $\mathrm{~F}$ & nd & - \\
\hline
\end{tabular}

reaction using the best IL in terms of yield and regioisomeric ratio. Consequently, the following three different set of reactions were carried out changing one of the reactive elements: palmitic acid, $\mathrm{KBr}$ and 1,2-hexanediol (1b), 1,2-pentanediol (1c), 1,2-butanediol (1d), 1,2-propanediol (1e) or 1,3-butanediol (1f). 1,2-Octanediol, $\mathrm{KBr}$ and acetic acid (2b), decanoic acid (2c), benzoic acid (2d), cinnamic acid (2e) or ibuprofen (2f). Palmitic acid, 1,2-octanediol and $\mathrm{KCl}, \mathrm{KI}$ or KF (Table 2).

Bromohydrin palmitate formation was observed for all the diols studied with moderate yields, $40-60 \%$ of isolated yield. Table 2 shows that the yield diminished as the carbon chain length of diol decreased. Nevertheless, bromohydrin ester with primary position halogen was always the main regioisomer. When the same reaction was carried out using other acids than palmitic, the yields also decreased. In this case, the regioisomeric ratio depends on the carboxylic acid used. Cinnamic acid yields the lowest regioisomeric ratio. However, ibuprofen yields a bromohydrin ester with a 80:20 (4k:5k) ratio and benzoic acid yields only $\mathbf{4 i}$, although the reaction yield was only $12 \%$. When $\mathrm{KCl}$ was used as the halide source, the halohydrin ester yield (55\% of isolated yield) was also moderate. However, a high regioselectivity, 80:20 (41:5I) ratio was also obtained. However, no reaction was observed in the presence of $\mathrm{KI}$ and $\mathrm{KF}$ as halide source.

To study some aspects of the mechanism of this transformation, two different reactions were carried out using palmitic acid, $\mathrm{KBr}$ and either 1,2-octanediol or 1-octanol as reagents. Their progress was followed by ${ }^{1} \mathrm{H}$ NMR. The formation and then the gradual decline of the two monopalmitates were observed in the first case. The highest yield of bromohydrin esters was observed after $48 \mathrm{~h}$ reaction. When 1-octanol was used, the formation of the corresponding octyl palmitate was also observed. However, no 1-bromooctane formation was detected after $48 \mathrm{~h}$ reaction. The ester was recovered unalterated in this second reaction. If we consider that the putative bromo derivative was only detected for 1,2octanediol, the presence of a diol system seems to be necessary to produce the halohydrin esters. The need for similar assistance has already been proposed by several authors to explain similar

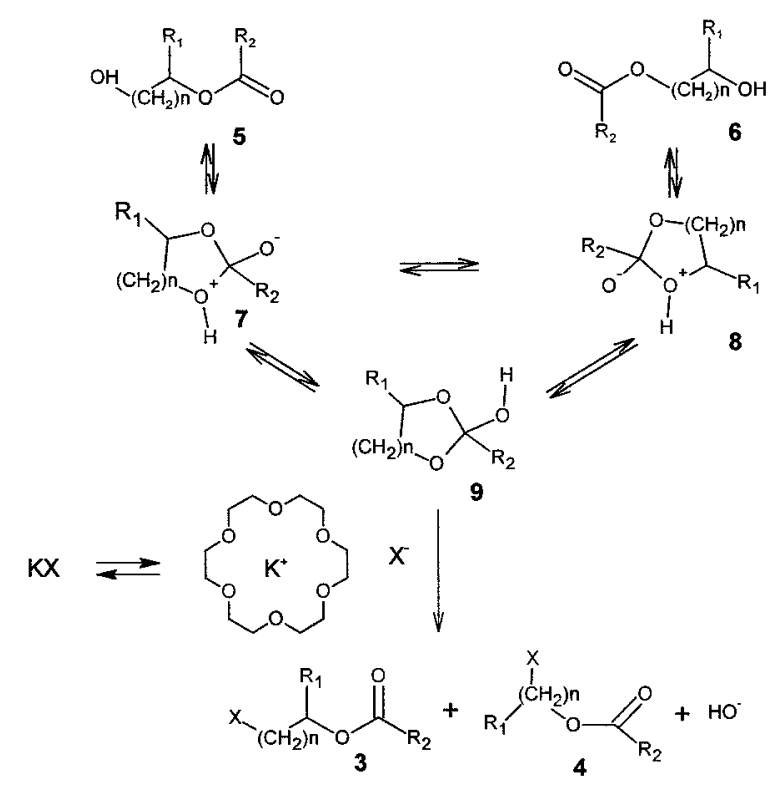

Fig. 2 Feasible mechanism scheme.

processes. ${ }^{17,24,25}$

A feasible mechanism is outlined in Fig. 2. Diol could be transformed into the monoesters followed by the formation of a dioxygenated ring $(\mathbf{7}, \mathbf{8})$, similar to those proposed by several

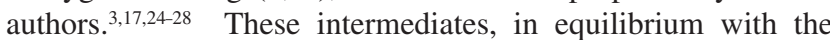
open monoester, can tautomerize to 9. Afterwards, this compound could react with halogen ions present in the medium through a nucleophilic opening process similar to the one proposed by several authors. ${ }^{17,24-27}$ Although the detailed catalytic function of ILs is not clear at this stage, the preliminary experimental results showed that bromoesterificaction in ILs is not only possible but also satisfactory. Moreover, polarity and $\mathrm{H}$-bonding capability of the IL could have a big influence in the reaction behavior. ${ }^{29}$ Polarity and $\mathrm{H}$-bonding are dependent on both anions and cations present in ILs.

\section{Conclusions}

We have developed a hopeful and greener one-pot methodology for obtaining halohydrin esters from commercial substances using $[\mathrm{BMIM}]\left[\mathrm{PF}_{6}\right]$ as reaction media and catalyst. $\mathrm{KX}$ can be used as a harmless halide source. The reaction is fairly regioselective depending on the halide used. We have shown that the described reaction can be carried out with diverse diols and acids. The application of this methodology to glycerol and other polyols is underway. These studies will be reported in due course.

\section{Acknowledgements}

The authors are grateful to the Ministerio de Educación y Ciencia for the FPU grant of Mireia Oromí Farrús and to the Ministerio de Educación y Ciencia (CTQ2006-07451) for financial support.

\section{References}

1. K. G. Watson, Y. M. Fung, M. Gredley, G. J. Bird, W. R. 
Jackson, H. Gountzos, and B. R. Matthews, Chem. Commun., 1990, 15, 1018.

2. J. Beger, J. Prakt. Chem., 1991, 333, 677.

3. H. C. Kolb and K. B. Sharpless, Tetrahedron, 1992, 48, 10515.

4. S. Hamaguchi, T. Ohashi, and K. Watanabe, Agric. Biol. Chem., 1986, 50, 375.

5. D. G. Talekar, P. L. Joshi, P. Ramaiah, and A. S. Rao, Indian J. Chem., Sect. B: Org. Chem. Incl. Med. Chem., 1986, $25,145$.

6. B. Damin, J. Garapon, and B. Sillion, French Patent Application, 1981, 2482086.

7. R. C. Larock, "Comprehensive Organic Transformations. A Guide to Functional Group Preparations", 1999, Wiley$\mathrm{VCH}, \mathrm{New}$ York.

8. M. Tanaka, M. Koyanagi, and T. Kobayashi, Tetrahedron Lett., 1981, 22, 3875.

9. S. D. Stamatov and J. Stawinski, Tetrahedron Lett., 2006 , 47, 2543.

10. R. K. Robert and B. Ganem, Tetrahedron Lett., 1974, 11, 913.

11. T. Oriyama, A. Ishiwata, Y. Hori, T. Yatabe, N. Hasumi, and G. Koga, Synlett, 1995, 10, 1004.

12. M. S. Newman and D. R. Olson, J. Org. Chem., 1973, 38, 4203.

13. S. M. Mcelvain and A. N. Bolstad, J. Am. Chem. Soc., 1951, 73, 1988.

14. S. Bhar and B. C. Ranu, J. Org. Chem., 1995, 60, 745.

15. T. Aoyama, T. Takido, and M. Kodomari, Tetrahedron Lett.,
2005, 46, 1989.

16. M. U. Ahmad, A. A. Ansari, and S. M. Osman, Fette, Seifen, Anstrichmittel, 1980, 82, 106.

17. J. Eras, J. J. Mendez, M. Balcells, and R. Canela, J. Org. Chem., 2002, 67, 8631.

18. T. Welton, Coord. Chem. Rev., 2004, 248, 2459.

19. H. Zhao and S. V. Malhotra, Aldrichimica Acta, 2002, 35, 75 .

20. L. W. Xu, L. Li, C. G. Xia, and P. Q. Zhao, Tetrahedron Lett., 2004, 45, 2435.

21. N. Jain, A. Kumar, S. Chauhan, and S. M. S. Chauhan, Tetrahedron, 2005, 61, 1015.

22. H. Olivier-Bourbigou and L. Magna, J. Mol. Catal. A: Chem., 2002, 182, 419.

23. L. F. Fieser and M. Fieser, "Reagents for Organic Synthesis", 1975, John Wiley and Sons, Inc., New York.

24. B. T. Golding, D. R. Hall, and S. Sakrikar, J. Chem. Soc., Perkin Trans. 1, 1973, 1214.

25. M. S. Newman and C. H. Chen, J. Am. Chem. Soc., 1973, 95, 278.

26. S. H. Wilen, L. Delguzzo, and R. Saferstein, Tetrahedron, 1987, 43, 5089.

27. M. S. Newman and C. H. Chen, J. Am. Chem. Soc., 1972, 94, 2149.

28. M. S. Ahmad, M. Khan, and S. M. Osman, Indian J. Chem., Sect. B: Org. Chem. Incl. Med. Chem., 1980, 19, 1001.

29. Z. Guo, B. Q. Chen, R. L. Murillo, T. W. Tan, and X. B. Xu, Org. Biomol. Chem., 2006, 4, 2772. 